\title{
Eficiência Técnica dos Gastos Públicos do Ensino Fundamental no Estado do Pará em 2017
}

\author{
Technical Efficiency of Public Elementary Expenditure in the State of Pará in 2017
}

\author{
Bruna de Souza Xavier*a; Juliana de Sales Silva ${ }^{\mathrm{b}}$ \\ ${ }^{a}$ Universidade Federal de Goiás, Programa de Pós-Graduação Stricto Sensu em Economia. GO, Brasil. \\ bUniversidade Federal do Sul e Sudeste do Pará. PA, Brasil. \\ *E-mail: bruninhaxavier@live.com.
}

\begin{abstract}
Resumo
Avaliar se os gastos com Educação são aplicados de maneira eficiente é de suma importância para possibilitar as tomadas de decisões na condução da aplicação dos recursos públicos. Por consequência, o objetivo desta pesquisa é analisar a eficiência técnica dos gastos públicos em Educação Fundamental no Estado do Pará no ano de 2017. Para tanto, é utilizada uma metodologia em dois estágios. No primeiro estágio é utilizada a técnica não paramétrica da Análise Envoltória de Dados (DEA), com orientação a output, estimação dos escores de eficiência técnica dos gastos públicos com Educação Fundamental dos municípios do Estado. No segundo estágio é utilizada uma regressão truncada para estimação dos determinantes dessa eficiência técnica. Os resultados do DEA indicaram que o gasto com Educação no município é o principal insumo que deve ser utilizado de forma ótima. Os resultados mais relevantes do segundo estágio apontaram que características como PIB municipal per capita, IDHM renda, população e saneamento tendem a elevar os níveis de eficiência, enquanto o FPM, mesmo sendo significativo, tende a diminuir a eficiência técnica dos municípios.
\end{abstract}

Palavras-chave: Educação. DEA. Regressão Truncada.

\begin{abstract}
Assessing whether education expenditures are applied efficiently is of paramount importance, to enable decision making in the conduct of the application of public resources. Consequently, the objective of this research is to analyze the technical efficiency of public spending on fundamental education in the state of Pará in the year 2017. To do so, a two-stage methodology is used. In the first stage, the non-parametric technique of Data Envelopment Analysis (DEA) is used, with an orientation to output, estimation of the technical efficiency scores of public spending on fundamental education in the state municipalities. In the second stage, a truncated regression is used to estimate the determinants of this technical efficiency. The DEA results indicated that spending on education in the municipality is the main input that should be used optimally. The most relevant results of the second stage pointed out that characteristics such as municipal GDP per capita, IDHM income, population and sanitation tend to increase the efficiency levels, while the FPM, although significant, tends to decrease the technical efficiency of the municipalities.
\end{abstract}

Keywords: Education. DEA. Truncated Regression.

\section{Introdução}

A Educação tem papel fundamental quando se trata da qualidade do bem-estar social, principalmente, quando os gastos públicos são utilizados de forma eficiente. O estado do bem-estar social é um tipo de institucionalização para tratar de desigualdades relacionadas à pobreza, ao desemprego, às vulnerabilidades econômicas e à incapacidade do trabalho (PRADO, 2013).

Por sua vez, a Educação influencia as condições de vida do indivíduo, promovendo melhor bem-estar para aqueles que a têm, mas, também, gerando uma série de externalidades, como, por exemplo, maior produtividade (MORETI, 2004). Do ponto de vista do desenvolvimento de um país, gera redução da pobreza e do desemprego e possibilita melhores expectativas para a vida social em seu mais diverso ponto de vista, como o aumento dos salários por meio da produtividade crescente. Além disso, maiores níveis educacionais impactam sobre o crescimento da renda per capita, trazendo consigo eficiência dos familiares em utilizar os recursos existentes e, consequentemente, uma melhor expectativa de vida maior (IPEA, 1997).

Para tais benefícios, há necessidade da ação do Estado, principalmente, no que se refere ao financiamento da Educação, visto garantir os recursos mínimos e assegurar a qualidade do ensino oferecido (SOBREIRA; CAMPOS, 2008).

No que tange aos gastos no setor educacional, o Brasil está entre os países que menos gasta com o Ensino Fundamental. A quantia média desembolsada por ano com cada estudante nessa fase escolar pelos países é de US\$ 8,7 mil, sendo Luxemburgo, na Europa, o primeiro da lista, gastando cerca de US\$21,2 mil, enquanto o Brasil gasta cerca de US\$ 3,8 mil por aluno no Ensino Fundamental. O setor público educacional nacional gasta 5,4\% do PIB, considerado, em relação aos países da América Latina, um gasto acima da média. No entanto, países como Colômbia (US\$ 2,5 mil) e México (US\$ 
2,9 mil) gastam menos por estudante do que o Brasil e têm melhores desempenhos nos testes do PISA (Programme for International Student Assessment, Programa Internacional de Avaliação de Estudantes) (OCDE, 2018).

Os últimos resultados do PISA de 2015, cuja coordenação é feita pela Organização para Cooperação e Desenvolvimento Econômico (OCDE), mostram que, entre os 70 países da OCDE participantes da avaliação, o Brasil obteve a $63^{\mathrm{a}}$ posição em Ciências, 59 $\mathrm{a}$ em Leitura e $66^{\mathrm{a}}$ colocação em Matemática (FUNDAÇÃO ABRINQ, 2017).

Este teste, comprovado pela OCDE, mostra como alunos de 15 anos aplicam seus conhecimentos no Ensino Educacional, o que pode ser interpretado como proxies de melhorias na eficiência dos gastos públicos.

Ao analisar o Norte do Brasil, Dias e Silva (2013) apontam que, com base nos dados da DEFP (Despesas com Educação Fundamental por População) de 2010, ocorre uma má aplicação dos recursos públicos na área da Educação em mais de $50 \%$ dos municípios. Além disso, no mesmo período, houve um declínio no nível educacional em termos de taxa de analfabetismo entre a população de 10 a 17 anos, tendo sido observada uma taxa de 5,4\%, a maior do País. Posteriormente, no ano de 2015, essa taxa aumentou para 9,2\% (FUNDAÇÃO ABRINQ, 2017), com diminuição em 2016 para 8,5\% (IBGE, 2017).

Com relação à aprendizagem das crianças, no ano de 2014, com base nos dados do MEC (Ministério da Educação), INEP (Instituto Nacional de Estudos e Pesquisas Educacionais Anísio Teixeira) e da DEEP (Diretoria de Estatísticas Educacionais), foram observados baixos percentuais em Leitura $(64,9 \%)$, Escrita (42,9\%) e Matemática (25,1\%) em mais de 50\% das crianças na região Norte, indicando baixo desempenho nessas competências e dificuldades das crianças do $3^{\circ}$ ano do Ensino Fundamental (FUNDAÇÃO ABRINQ, 2017).

No que se refere à taxa de distorção idade-série ${ }^{1}$ dos alunos do Ensino Fundamental, que é quando ocorre situação de atraso escolar, são verificadas, na Região supracitada, as mais altas taxas de distorção $(28,3 \%)$, ultrapassando a taxa nacional de 19,2\%, segundo relatório da Fundação Abrinq (2017).

Em relação ao Estado do Pará, foram verificadas, nos municípios do Estado, as maiores despesas no Ensino Fundamental no ano de 2005 por aluno, destacando-se Anapu, com uma DEFP de R\$423,33; em 2007, o município de Afuá, com R\$ 552,69; e em 2009, Juruti, com R\$ 990,23. No que se refere aos municípios com menores despesas: em 2005, Capitão Poço com o menor DEFP, de R\$ 26,08; em 2007, Ananindeua, com R\$ 61,83; e em 2009, Água Azul do Norte, com R\$ 91,36 (DIAS; SILVA, 2013).

No que se refere ao desempenho de aprendizagem no Estado no ano de 2015, verificou-se um percentual de
$18 \%$, sendo Matemática a disciplina em que ocorreu o pior desempenho. Além disso, o Pará, neste mesmo ano, obteve a menor pontuação no Índice de Desenvolvimento da Educação Básica - IDEB, média de 4,5 (FUNDAÇÃO ABRINQ, 2017).

Ressalva-se então que o baixo desempenho do Ensino Público do Pará pode ter sido causado pela má aplicação de recursos públicos, que podem não estar sendo utilizados de maneira eficiente. Nesse sentido, observa-se que estudos que tratam da averiguação de como são feitos os gastos públicos com o ensino no Pará são importantes, uma vez que podem dar indícios de como esses podem ser alocados de forma correta junto às políticas públicas. Portanto, é de suma importância uma averiguação de como são alocados os gastos públicos com o Ensino Fundamental no Estado, se esta alocação é feita de forma eficiente e, caso não seja, quais alterações seriam necessárias para alcançar a tal eficiência.

Sendo assim, este trabalho tem como objetivo geral analisar a eficiência técnica dos gastos públicos de Educação Fundamental no Estado do Pará em 2017. Para atingir tal objetivo se pretende estimar os escores de eficiência, empregando o método não paramétrico de Análise Envoltória de Dados (DEA) e, posteriormente, analisar os possíveis determinantes da eficiência técnica, sendo utilizada uma regressão truncada.

Na literatura, diversos trabalhos já analisaram a eficiência técnica de gastos com Educação. No âmbito internacional, podem ser citados os trabalhos de Ruggiero et al. (1999), Afonso e St. Aubyn (2005) e Alexander e Jang (2017). Já nacionalmente se têm os trabalhos de Zoghbi et al. (2009), Diniz e Corrar (2011), Machado Júnior et al. (2011), Zoghbi et al. (2011), Almeida e Gasparini (2011), Silva e Almeida (2012), Savian e Bezerra (2013), Santos et al. (2017) e Lourenço et al. (2017). Para a região Norte se tem o trabalho de Dias e Silva (2013) e, especificamente, para o Estado do Pará, foi encontrado, até o momento, apenas o trabalho de Rodrigues et al. (2018).

Dias e Silva (2013) tiveram como objetivo, em pesquisa, analisar se os recursos gastos no Ensino Fundamental pela gestão pública de 371 municípios da Região Norte do Brasil têm sido utilizados de forma eficiente para os anos 2005, 2007 e 2009. Para tal, os autores utilizaram uma metodologia baseada em Brunet et al. (2007), denominada de Escore Padronizado pelo Método da Função Distribuição Acumulada Normal, para calcular o IQGP (Índice de Qualidade dos Gastos Públicos), com base no Índice de Bem-Estar e Índice de Insumo. Os resultados mostraram que na região Norte do Brasil não houve eficiência na aplicação dos recursos públicos na área da educação em mais de $50 \%$ dos municípios. No entanto, a hipótese da pesquisa de que não houve eficiência na aplicação dos recursos públicos na área da educação não foi comprovada, pois a maioria dos municípios apresentou

1 Proporção de alunos com mais de dois anos de atraso escolar, ou seja, com idade maior do que a faixa etária-limite prevista para cada um dos níveis de ensino (FUNDAÇÃO ABRINQ, 2017). 
IQGP superior a um, o que, possivelmente, pode significar que houve eficiência na gestão da execução dos orçamentos educacionais.

O trabalho de Rodrigues et al. (2018) buscou avaliar esta eficiência por meio da metodologia DEA com orientação input para 144 municípios do Estado para o ano de 2010. Entre os principais resultados dos autores, 19 municípios alcançaram a eficiência técnica máxima, mas a maioria dos municípios não está utilizando os recursos para educação de forma eficiente.

Percebe-se que, apesar de haver uma vasta literatura sobre as análises de eficiência técnica de gastos com educação, poucas análises se reportam aos determinantes dessa eficiência. Sendo assim, pretende-se com esta pesquisa suprir tal lacuna encontrada até o momento. Portanto, este trabalho tem como objetivo geral analisar a eficiência técnica dos gastos públicos de Educação Fundamental no Estado do Pará em 2017. Para atingir tal objetivo, pretende-se estimar os escores de eficiência, empregando o método não paramétrico de Análise Envoltória de Dados (DEA) e, posteriormente, analisar os possíveis determinantes da eficiência técnica, sendo utilizada uma regressão truncada.

\section{Material e Métodos}

Para as análises de eficiência podem ser adotados dois tipos de métodos: os não paramétricos e os paramétricos. Para alcançar seus objetivos, analisar a eficiência técnica dos gastos públicos com Ensino Fundamental nos municípios do Pará e seus determinantes, foi utilizada uma abordagem em dois estágios. No primeiro estágio foi estimada a eficiência por meio da Análise Envoltória de Dados, DEA (Data Envelopment Analysis), e no segundo, os determinantes de tal eficiência.

\subsection{Primeiro estágio: Data Envelopment Analysis (DEA)}

Segundo Ferreira e Gomes (2009), DEA é um método quantitativo, empírico, não paramétrico, que não se submete às condições parametrizadas das análises estatísticas e econométricas, que avalia o desempenho de organização ou de atividades denominadas de DMUs (Decision Making Units). As análises são feitas por comparação entre as DMUs, segundo a qual a eficiência é dada pela relação entre inputs utilizados e outputs gerados. Assim, quanto maior a produção de uma DMU para uma dada quantidade de insumos, ou, quanto menor a quantidade de insumos utilizada pela DMU para uma determinada quantidade de produto, maior será sua eficiência.

Tecnicamente, a metodologia DEA usa a programação matemática linear para estimar a fronteira de possibilidades de produção com as unidades eficientes, o que permite identificar as DMUs tomadas como padrão para as demais unidades avaliadas, isto é, aquelas unidades que ficam posicionadas na fronteira (DMUs eficientes) e as que se posicionam abaixo da fronteira de possibilidade de produção (DMUs ineficientes)

\section{(GOMES; BAPTISTA 2004).}

A medida de eficiência para cada DMU é obtida pela razão entre a soma ponderada dos produtos e a soma ponderada dos insumos. Para a $i$-ésima DMU, tem-se:

Eficiência da DMU $i=\frac{\mu^{\prime} y_{i}}{v \prime x_{i}} \frac{\mu_{1} y_{1 i}+\mu_{2} y_{2 i}+\ldots+\mu_{m i} y_{m i}}{v_{1} x_{1 i}+v_{2} x_{2 i}+\ldots+v_{k i} x_{k i}}=(1)$

em que $\mu$ é um vetor ( $\mathrm{m} \times 1$ ) de pesos associados aos produtos; $v$ é um vetor ( $\mathrm{k} \times 1)$ de pesos associados aos insumos; $y$ se refere aos produtos; e $x$, aos insumos utilizados na $i$-ésima DMU.

O modelo DEA pode ser de dois tipos: CCR, proposto por Charnes, Cooper e Rhodes em 1978, que utiliza o axioma da proporcionalidade entre inputs e outputs; e o BCC, proposto por Banker, Charnes e Cooper em 1984, que utiliza o axioma da convexidade (FERREIRA; GOMES, 2009). Diante disto, utiliza-se abordagem BCC neste trabalho, com orientação output (produto=nota média do IDEB) com retornos variáveis de escala. De acordo com Mello et al. (2005), este modelo pode ser especificado da seguinte maneira:

Sujeito à:

$$
\begin{aligned}
& \operatorname{Max} E f_{\theta}=\left(\sum_{j=1}^{M} \mu_{j} y_{j \theta}\right)+u_{i} \\
& \sum_{i=1}^{R} v_{i} x_{i \theta}=1 \\
& \sum_{j=1}^{M}\left(\mu_{j} y_{j \theta}+u_{i}\right) \leq \sum_{i=1}^{R} v_{i} x_{i \theta}=\leq 0, j=1, \ldots, n
\end{aligned}
$$

\subsection{Segundo estágio: determinantes de eficiência}

Após a estimação dos escores de eficiência, o segundo estágio pode ser alcançado pela abordagem paramétrica à regressão censurada (Tobit).

De acordo com Gomes (2010), o segundo estágio de estimação leva em conta variáveis como fixas ou não discricionárias, cujo sinal dos coeficientes indica a direção da influência que pode ser empregada para avaliar o nível de significância desses coeficientes.

Com base em Greene (1981), o modelo de regressão Tobit pode ser expresso por:

$$
\theta=\alpha_{0}+\mu_{i} \beta+\varepsilon_{i}
$$

em que $\theta$ é a eficiência obtida pela metodologia DEA; $\alpha_{0}$ $\alpha_{0}$ é uma constante; $u i$ é um vetor de variáveis explicativas, apresentados no Quadro 2 da próxima subseção; $\beta \beta$ é um vetor de parâmetros a ser estimado; e $\varepsilon_{i}$ é o erro de regressão. $O$ valor da eficiência será $0<\theta \leq 10<\theta \leq 1$.

De acordo com Greene (2002), este modelo é utilizado quando a variável dependente se encontra entre valores ou concentrada em pontos iguais a um valor-limite, isto é, censurada. Sendo assim, a utilização do Tobit visa contornar o problema da censura, valendo-se de técnicas estatísticas que possibilitem fazer inferências para toda a população sem perda de qualidade, como aconteceria no caso de variáveis 
truncadas, isto é, variáveis que apresentam concentração de pontos com valores iguais a um valor limite, comumente zero.

No entanto, Simar e Wilson (2007) apontam que a equação (3) apresenta algumas deficiências, como, por exemplo, resultados enviesados, em razão da correlação entre o termo de erro da regressão e as variáveis explicativas que a censura causa. Nesse sentido, aqui foram utilizadas as contribuições de Simar e Wilson (2007), bem como as de Badunenko e Tauchmann (2018), que sugerem estimações robustas, por meio do procedimento de bootstrap, isto é, por repetições para a obtenção de estimativas dos parâmetros, desvios padrão e intervalos de confiança.

Os dados utilizados, bem como a fonte para o primeiro estágio das estimativas, a Análise Envoltória de Dados estão expostos no Quadro 1.
Quadro 1 - Variáveis e fonte de dados do primeiro estágio

\begin{tabular}{|c|c|c|c|}
\hline Variável & Descrição & Ano & Fonte \\
\hline \multicolumn{4}{|c|}{ Input } \\
\hline Escolas & Número de escolas no município & 2017 & INEP \\
\hline Docentes & Número de docentes no município & 2017 & INEP \\
\hline Turmas & Número de turmas no município & 2017 & INEP \\
\hline Gastos & $\begin{array}{c}\text { Gastos totais com educação no } \\
\text { município }\end{array}$ & 2017 & FINBRA \\
\hline \multicolumn{3}{|c|}{ Output } \\
\hline $\begin{array}{c}\text { Nota } \\
\text { média do } \\
\text { IDEB }\end{array}$ & $\begin{array}{c}\text { Nota do IDEB 5 } \\
\text { IDEB } 9^{\circ} \text { ano }+ \text { Nota do } / 2\end{array}$ & 2017 & INEP \\
\hline
\end{tabular}

Fonte: Dados da pesquisa.

No que se refere aos dados e fontes do segundo estágio, é importante destacar que a variável dependente utilizada é o escore de eficiência estimado no modelo DEA, enquanto as variáveis independentes utilizadas estão expostas no Quadro 2 e foram selecionadas com base em Santos et al. (2015).

Quadro 2 - Variáveis e fonte de dados do segundo estágio

\begin{tabular}{|l|l|c|c|c|}
\hline \multicolumn{1}{|c|}{ Variável } & \multicolumn{1}{|c|}{ Descrição } & Código & Ano & Fonte \\
\hline PIB municipal per capita & Produto Interno Bruto municipal per capita & lnpib_pc & 2015 & IBGE \\
\hline FPM & Repasses do Fundo de Participação do Município & lnfpm & 2017 & Tesouro Nacional \\
\hline População & População total do município & lnpop & 2010 & IBGE \\
\hline IDHM Renda & $\begin{array}{l}\text { Índice de Desenvolvimento Humano Municipal } \\
\text { relativo à Renda }\end{array}$ & idhm_renda & 2010 & Atlas Brasil \\
\hline Saneamento & $\begin{array}{l}\text { Número de domicílios com banheiro ou sanitário e } \\
\text { esgotamento sanitário, água canalizada, coleta de lixo e } \\
\text { energia elétrica }\end{array}$ & Saneamento & $\begin{array}{c}2010 \\
\text { Censo Demográfico/ } \\
\text { IBGE }\end{array}$ & \begin{tabular}{l} 
Sala \\
\hline
\end{tabular}
\end{tabular}

Fonte: Dados da pesquisa.

No levantamento de dados dos municípios que compõem o Estado do Pará, verificou-se que, dos 144 municípios, $22^{1}$ não disponibilizaram informações suficientes para a estimação dos modelos aqui utilizados, desta forma, tais municípios foram retirados da amostra.

\section{Resultados e Discussão}

Primeiramente, será exposta a análise descritiva dos dados aqui utilizados. Posteriormente, serão apresentados os resultados da Análise Envoltória de Dados (DEA), por fim, os resultados da Regressão Truncada.

\subsection{Análise descritiva}

Para caracterizar a amostra utilizada nesta pesquisa, foram feitas algumas análises descritivas das variáveis, como as médias, desvios padrão e os valores máximos e mínimos (Quadro 3).

Quadro 3 - Estatísticas descritivas das variáveis utilizadas

\begin{tabular}{|c|c|c|c|c|}
\hline Variável & Média & Desvio Padrão & Mínimo & Máximo \\
\hline Nota IDEB & 4,1 & 0,8 & 3,0 & 5,4 \\
\hline Gastos $(\mathrm{R} \$)$ & $34.860 .745,61$ & $42.401 .627,25$ & $861.233,00$ & $342.128 .312,07$ \\
\hline Docentes $\left(\mathrm{n}^{\circ}\right)$ & 412 & 518 & 34 & 4.509 \\
\hline Escolas $\left(\mathrm{n}^{\circ}\right)$ & 63 & 57 & 5 & 402 \\
\hline Turmas $\left(\mathrm{n}^{\circ}\right)$ & 422 & 533 & 36 & 4.493 \\
\hline PIB per capita (R\$) & $12.710,52$ & $11.480,65$ & $4.945,00$ & $103.806,00$ \\
\hline FPM (R\$) & $19.993 .984,29$ & $33.382 .363,95$ & $5.364 .510,00$ & $360.160 .568,23$ \\
\hline População & 57.705 & 137.699 & 3.431 & 1.393 .399 \\
\hline IDHM Renda & 0,576 & 0,059 & 0,449 & 0,751 \\
\hline Saneamento & 14.038 & 35.625 & 981 & 368.889 \\
\hline
\end{tabular}

Nota: Os valores de Docentes, Escolas e Turmas foram arredondados.

Fonte: Dados da pesquisa.

2 Altamira, Bagre, Bonito, Breves, Cachoeira do Arari, Curralinho, Faro, Ipixuna do Pará, Jacundá, Marapanim, Mojuí dos Campos, Muaná, Porto de Moz, Salvaterra, Santarém Novo, Santo Antônio do Tauá, São Caetano de Odivelas, São Domingos do Capim, São Geraldo do Araguaia, São João do Araguaia, Terra Alta, Vitória do Xingu. 
Nas informações dispostas no Quadro 3, observa-se que a nota média do IDEB foi 4,1 no Ensino Fundamental, resultado abaixo da meta 5 para o ano de 2017. Tal resultado evidencia a necessidade de melhoria na qualidade do Ensino Fundamental no Estado do Pará. De acordo com o IDEB (2017), a meta para a nota nacional no ano de 2022 é 6,0, atual patamar educacional da média dos países da OCDE (IDEB, 2017).

No que se refere à média dos gastos com Educação Fundamental por municípios, o gasto médio foi de $\mathrm{R} \$$ $34.860 .745,61$, média de baixa. No ano de 2017, o Fundo Nacional da Educação teve que repassar R \$ 918,97 milhões de recursos a nove Estados, que não tinham verba educacional, incluindo o Pará. Tal recurso foi repassado em concordância com a Lei $n^{\circ}$ 11.494/ 2007, que instituiu o FUNDEB, a qual informa que os Estados que não alcançarem a arrecadação do valor mínimo por gastos por alunos de R $\$ 2.857,03$ devem ter um repasse para complementar a despesa (BRASIL, 2017).

Outras variáveis que compõem os insumos utilizados aqui foram: número de docentes, que obteve média de 412; número de turmas, que obteve uma média 422; e número de escolas, que foi de 63. Conforme o Artigo $5^{\circ}$ da Instrução Normativa $\mathrm{N}^{\mathrm{o}}$ 3, de 1 de março de 2016, a lotação de professores nas escolas públicas estaduais deve ser feita em consonância com o número de turmas ofertadas (PARÁ, 2016). Portanto, as médias encontradas mostram que a Instrução Normativa é atendida relativamente à média dessas variáveis, atendendo ao requisito, conforme instrução acima. Além disso, observase alto valor do desvio padrão nessas variáveis, indicando heterogeneidade na amostra.

No que se refere à média do PIB municipal per capita, o valor encontrado foi de R\$12.710,52, já para a população a média foi de $\mathrm{R} \$$ 57.705. A influência da variável PIB per capita na riqueza dos municípios ocorre em função do incremento do padrão de vida da população, contudo as médias descritas não consideram o nível de desigualdade de renda da sociedade paraense, pois não expressam importantes fatores, como distribuição de renda, qualidade de vida, educação e saúde (CAETANO et al., 2019). Por sua vez, a média do FPM (Fundo de Participação dos Municípios) foi de R\$19.993.984,29 transferência dos recursos aos municípios, feita segundo a faixa populacional. Utiliza-se para a distribuição do FPM um coeficiente que varia de 0,6 a 4,0, com o número de habitantes entre 10.188 e 156 mil ou acima, de acordo com a Lei n ${ }^{\circ}$ 5.172, de 25 de outubro de 1966 (BRASIL, 1966). O Fundo de Participação dos Municípios tem como objetivo reduzir as disparidades econômicas entre esses.

Analisando a variável do IDHM rendase percebe que a média foi de 0,576 , enquadrando-se em uma faixa baixa, isto é, os municípios paraenses, em média, têm baixo acesso às necessidades básicas, como: água, comida e abrigo, impedindo uma maior oportunidade de viver uma vida longa e saudável, de ter acesso ao conhecimento e de ter um padrão de vida que garanta as necessidades básicas.

Sobre a variável proxy de saneamento, que se refere aos domicílios que têm banheiro ou sanitário e esgotamento sanitário, água canalizada, coleta de lixo e energia elétrica, a média é de 14.038 , o equivalente a $26 \%$ do máximo alcançado, o que pode influenciar no aproveitamento dos alunos no ensino escolar, dado que sem acesso ao saneamento básico, como abastecimento de água potável, esgotamento sanitário, limpeza urbana e manejo de resíduos sólidos, há inviabilidade de realização de atividades escolares, além de uma redução na proficiência escolar. Além disso, é na infância que está o maior número de vítimas de doenças relacionadas à falta de saneamento, implicando o desenvolvimento da criança no futuro (TRATA BRASIL, 2008).

\subsection{Resultados do primeiro estágio: DEA}

O Quadro 4 destaca a eficiência técnica dos gastos municipais do Pará em 2017, sob condição de retornos constantes e variáveis de escala. Aqui, foram considerados eficientes os municípios que alcançaram escores de eficiência 1 e ineficientes os que obtiveram níveis abaixo desse valor. No Quadro 4, podem ser observadas as médias, valores máximos e mínimos, desvios padrão, quantidade de municípios eficientes para retornos constantes, variáveis e com eficiência de escala.

Quadro 4 - Eficiência técnica dos gastos municipais do Pará em 2017, sob condição de retornos constantes e variáveis de escala

\begin{tabular}{|l|c|c|c|}
\hline $\begin{array}{c}\text { Escore de } \\
\text { eficiência }\end{array}$ & $\begin{array}{c}\text { Retornos } \\
\text { Constantes }\end{array}$ & $\begin{array}{c}\text { Retornos } \\
\text { Variáveis }\end{array}$ & $\begin{array}{c}\text { Eficiência de } \\
\text { Escala }\end{array}$ \\
\hline Média & 0,737 & 0,761 & 0,969 \\
\hline Máximo & 1 & 1 & 1 \\
\hline Mínimo & 0,528 & 0,556 & 0,828 \\
\hline Desvio padrão & 0,114 & 0,114 & 0,036 \\
\hline $\begin{array}{l}\text { Municípios } \\
\text { eficientes }\end{array}$ & 4 & 7 & 7 \\
\hline \% da amostra & 3,3 & 5,7 & 5,7 \\
\hline
\end{tabular}

Fonte: Dados da pesquisa.

No Quadro 4, quando são observados os retornos constantes, verifica-se que, do total dos 122 municípios, apenas quatro tiveram eficiência técnica, o que corresponde à baixa porcentagem de 3,3\% do total da amostra, evidenciando a necessidade de melhoria na eficiência dos gastos municipais do Pará no Ensino Fundamental. Tais informações são importantes para que políticas públicas sejam inseridas para elevar o nível de eficiência dos municípios paraenses, como, por exemplo, políticas que assegurem o ingresso mais longo para a aprendizagem da alfabetização e letramento. Por sua vez, a média do escore de eficiência foi de 0,737 , mostrando uma aproximação do escore 1 de eficiência, enquanto sua ineficiência técnica no modelo CCR, quando calculada (10,737 ), ficou em 0,263, indicando necessidade de reduzir em $26,30 \%$ os gastos municipais paraenses, ou seja, usar menos insumos (inputs), mantendo, assim, o mesmo nível de produção para alcançar a eficiência técnica.

Considerando os escores de eficiência sob a ótica dos retornos variáveis, observa-se que a eficiência técnica média obteve pequeno aumento, 0,761, comparativamente aos retornos constantes. Essa diferença indica mais três municípios 
eficientemente técnicos, totalizando sete, o que corresponde a $5,7 \%$ da amostra. Portanto, nota-se um pequeno crescimento na média dos municípios eficientes, considerando o modelo BCC, quando se deixa de analisar retornos constantes para analisar os retornos variáveis. Esta evidência é explicada, segundo Gomes e Baptista (2004), em razão de os valores obtidos para a eficiência técnica no modelo BCC serem maiores do que no modelo CCR, assim, se uma DMU é eficiente no modelo CCR, será também eficiente no modelo BCC.

O Quadro 4 apresenta também os resultados da eficiência de escala, que é a relação entre os dois modelos de eficiência técnica, o CCR e o BCC, ou seja, quando os gastos são gerenciados em escala ótima, os municípios serão considerados eficientes. Observa-se que sete municípios se mostraram eficientes, com um escore médio de eficiência de 0,969 , o equivalente a 5,7\% dos municípios do Pará, produzindo em escala ótima de produção.

Parte-se agora para a distribuição dos gastos municipais de acordo com a escala de produção, Quadro 5, que mostra o grau em que um aumento proporcional em todas as quantidades de insumos aumenta as quantidades de produtos.

Quadro 5 - Distribuição dos gastos municipais do Pará em 2017, segundo o tipo de retorno

\begin{tabular}{|l|c|c|}
\hline \multicolumn{1}{|c|}{ Escala de produção } & $\mathbf{N}^{\mathbf{0}}$ Municípios & $\mathbf{\%}$ \\
\hline Retornos crescentes & 65 & 53,3 \\
\hline Retornos constantes & 6 & 4,9 \\
\hline Retornos decrescentes & 51 & 41,8 \\
\hline Total & 122 & 100 \\
\hline
\end{tabular}

Fonte: Dados da pesquisa.
O Quadro 5 mostra que, do total de municípios do Pará, 65 estão com escala de produção com retornos crescentes, o que significa que $53,3 \%$ da amostra poderia melhorar eficientemente, tendo grande escala de produção nos municípios, pois estes municípios operam com baixos valores de insumos, podendo expandir para se tornarem eficientes.

Quanto aos retornos constantes, que é quando existe uma proporcionalidade entre produto e insumos, seis municípios se enquadraram nesta escala de produção, isto é, 4,9\% da amostra. Por último, 51 municípios apresentaram retorno decrescente à escala, o que corresponde a $41,8 \%$ do total da amostra, indicando que o nível de eficiência seria maior com o aumento da produção e em uma proporção menor de inputs, pois, neste caso, a proporção total do produto é menor que a proporção dos insumos.

O Quadro 6 apresenta os resultados do modelo DEA, contendo os sete municípios mais eficientes e os sete menos eficientes do Pará, em relação aos gastos municipais em $2017^{3}$. Os sete municípios mais eficientes serviram de benchmarks ${ }^{4}$ para os sete municípios menos eficientes ${ }^{5}$. Observa-se também que no Quadro 6 estão inseridas as notas do IDEB, os números de docentes, de escolas e de turmas, bem como os gastos para utilização dos municípios. Os valores entre parênteses dos sete municípios menos eficientes representam os valores que deveriam ser diminuídos para que esses se tornem eficientes, tendo como base os benckmarks.

Quadro 6 - Comparação dos sete municípios mais eficientes com os sete menos eficientes, gastos municipais do Pará em 2017

\begin{tabular}{|c|c|c|c|c|c|c|c|c|c|}
\hline Ranking & Escore & DMU & Benchmarks & $\begin{array}{l}\text { Nota } \\
\text { média } \\
\text { IDEB }\end{array}$ & $\begin{array}{c}\text { Docente } \\
\left(\mathbf{n}^{\circ}\right)\end{array}$ & $\begin{array}{c}\text { Escolas } \\
\left(\mathbf{n}^{0}\right)\end{array}$ & $\begin{array}{c}\text { Turmas } \\
\left(\mathbf{n}^{\circ}\right)\end{array}$ & Gastos (RS) & Retorno \\
\hline $1^{\circ}$ & 1 & Abel Figueiredo & - & 4,4 & 47 & 8 & 50 & $7.424 .072,89$ & Decrescente \\
\hline $2^{\circ}$ & 1 & Bannach & - & 4 & 34 & 7 & 36 & $4.553 .646,81$ & Constante \\
\hline $3^{0}$ & 1 & Benevides & - & 5,2 & 341 & 40 & 366 & $15.224 .745,64$ & Decrescente \\
\hline $4^{\circ}$ & 1 & Santa Maria do Pará & - & 3,8 & 206 & 55 & 202 & $861.233,42$ & Constante \\
\hline $5^{\circ}$ & 1 & Santarém & - & 5 & 2597 & 402 & 2834 & $1.681 .482,84$ & Decrescente \\
\hline $6^{\circ}$ & 1 & Sapucaia & - & 3,7 & 51 & 5 & 44 & $7.768 .459,80$ & Constante \\
\hline $7^{\circ}$ & 1 & Ulianópolis & - & 5,4 & 170 & 28 & 180 & $21.218 .611,30$ & Decrescente \\
\hline \multirow{2}{*}{0,5926} & \multirow{2}{*}{ Chaves } & \multirow{2}{*}{$5^{\mathrm{o}} ; 7^{\mathrm{o}}$} & \multirow{2}{*}{3,2} & & 268 & 99 & 342 & $21.995 .599,00$ & Crescente \\
\hline & & & & & -58 & -42 & -96 & $-460.438,00$ & \\
\hline \multirow{2}{*}{$117^{\circ}$} & \multirow{2}{*}{0,5833} & \multirow{2}{*}{ Anajás } & \multirow{2}{*}{$5^{\circ} ; 7^{\circ}$} & \multirow{2}{*}{3,2} & 440 & 102 & 490 & $34.550 .226,56$ & Crescente \\
\hline & & & & & -158 & -43 & -181 & $-7.776 .796,00$ & \\
\hline \multirow{2}{*}{$118^{\circ}$} & \multirow{2}{*}{0,5741} & \multirow{2}{*}{ Cachoeira do Piriá } & \multirow{2}{*}{$7^{\circ}$} & \multirow{2}{*}{3,1} & 227 & 50 & 207 & $23.974 .461,26$ & Crescente \\
\hline & & & & & -33 & -13 & -16 & $-1.582 .057,00$ & \\
\hline \multirow{2}{*}{$119^{\circ}$} & \multirow{2}{*}{0,5741} & \multirow{2}{*}{ Gurupá } & \multirow{2}{*}{$7^{\circ}$} & \multirow{2}{*}{3} & 510 & 91 & 480 & $47.067 .664,18$ & Crescente \\
\hline & & & & & -195 & -36 & -172 & $-14.800 .000,00$ & \\
\hline \multirow{2}{*}{$120^{\circ}$} & \multirow{2}{*}{0,5648} & \multirow{2}{*}{$\begin{array}{c}\text { Nova Esperança do } \\
\text { Piriá }\end{array}$} & \multirow{2}{*}{$5^{\mathrm{o}} ; 7^{\mathrm{o}}$} & \multirow{2}{*}{3,1} & 248 & 48 & 226 & $21.266 .993,13$ & Crescente \\
\hline & & & & & -44 & -11 & -26 & $-27.327,50$ & \\
\hline \multirow{2}{*}{$121^{\circ}$} & \multirow{2}{*}{0,5648} & \multirow{2}{*}{ Almeirim } & \multirow{2}{*}{$5^{\mathrm{o}} ; 7^{\mathrm{o}}$} & \multirow{2}{*}{3,1} & 432 & 68 & 499 & $33.439 .627,74$ & Crescente \\
\hline & & & & & -148 & -23 & -180 & $-6.902 .612,00$ & \\
\hline \multirow{2}{*}{$122^{\circ}$} & \multirow{2}{*}{0,5555} & \multirow{2}{*}{ Afuá } & \multirow{2}{*}{$1^{\mathrm{o}} ; 5^{\mathrm{o}} ; 7^{\mathrm{o}}$} & 3 & 637 & 116 & 579 & $43.722 .204,37$ & Crescente \\
\hline & & & & 3 & -259 & -49 & -222 & $-12.500 .000,00$ & \\
\hline
\end{tabular}

Nota: Os valores entre parênteses representam os valores que deveriam ser diminuídos para que os municípios se tornassem eficientes, tendo como base os benckmarks.

Fonte: Dados da pesquisa.

3 A lista com todos os municípios está exposta no Apêndice 1.

$4 \mathrm{O}$ termo pode ser entendido como referência.

5 Ressalta-se que os mais eficientes serviram de referência para todos os ineficientes, mas para melhor visualização dos resultados, só são apresentados 14 municípios, para mais detalhes, ver Apêndice 1 
Diante disso, um dos maiores benefícios do uso da DEA é o conjunto de unidades de referência que pode ser usado como benckmarks na melhoria do desempenho das unidades menos eficientes, ou seja, indicam o que precisa ser alterado nos insumos e como aperfeiçoá-los para transformar unidades ineficientes em eficientes.

Entre os sete municípios eficientes, os que apresentaram retornos constantes à escala foram Bannach, Santa Maria do Pará, Sapucaia, indicando que têm a melhor relação produto/insumo e estão situados sobre a fronteira eficiente, cuja produção dobra quando há duplicação dos insumos. Os municípios que apresentaram retornos decrescentes de escala foram Abel Figueiredo, Benevides, Santarém, Ulianópolis, informando que o uso de insumos, como docentes escolas, turmas e gastos, aumenta, chegando a um ponto em que as quantidades adicionais de produtos obtidas se tornam menores, o que comprova maior variação dos insumos do que de produtos, pois, ao multiplicar os insumos, o produto sofrerá uma variação menor.

Com base nos resultados, percebe-se que Abel Figueiredo foi um município considerado eficiente, alcançando nota média do IDEB de 4,4. Este resultado está acima da meta projetada para o município para o ano de 2017 , de 4,05 , segundo INEP (2018), evidenciando um aumento da nota escolar dos alunos do Ensino Fundamental. Este resultado mostra um possível domínio crescente dos conteúdos, competências e habilidade desejáveis para ano/série subsequente do $5^{\circ}$ ao $9^{\circ}$ ano, contendo 47 docentes, oito escolas com 50 turmas e gastos de R\$ 7.424.072,89.

Bannach alcançou nota média 4 no IDEB, com 34 docentes, sete escolas, 36 turmas e gastos de R\$ 4.553.646,81. Os insumos e o produto são proporcionais, sem necessidade de reduzir ou expandir qualquer insumo, alcançando alto nível de eficiência. Benevides alcançou a segunda maior nota média do IDEB, 5,2, com 341 docentes, 40 escolas, 366 turmas e gastos de R\$ 15.224.745,64. Este resultado, possivelmente, esteja correlacionado ao fato de o município estar situado na região metropolitana de Belém, que teve evolução no seu desempenho educacional, principalmente depois da entrada do Programa "Benevides à Escola", implantado em 2013, que possibilitou acesso ao Ensino Educacional, com uma organização sistemática e uma identidade educacional, que antes o município não tinha (ENAP, 2013).

Santa Maria do Pará, Santarém e Sapucaia alcançaram nota média do IDEB de 3,75; 4,95 e 3,65, respectivamente. Entre esses municípios, Santarém se destaca, além da nota do IDEB, pela quantidade enorme de docentes, 2.597, necessários para a grande quantidade de turmas, 2.834, para um total de 302 escolas, com um gasto de R $\$ 1.681 .482,84$. Faz-se necessário avaliar que o município de Santarém, em termos educacionais, oferta jornada escolar integral, com ampliação dos conteúdos e do tempo, além do "Programa Mais Educação", iniciado em 2009, em dezoito escolas urbanas, o que influenciou o aumento dos níveis educacionais, além de ter um gasto relativamente baixo em relação ao número alto de docentes, turmas e escolas (FERREIRA; COLARES, 2017). Dessa forma, o município fez uma alocação eficiente de seus recursos, com mais acesso ao ensino.

Entre os municípios eficientes, a maior nota média do IDEB, 5,4, foi para Ulianópolis, que teve o maior gasto educacional, R\$21.218.611,30. Este município, localizado na Região do Sudeste paraense, foi um dos primeiros a aderir no ano de 2015 ao "Pacto Pela Educação no Pará", no intuito de alterar os índices da educação e aumentar em 30\% a nota do IDEB até 2017 (XERFAN, 2018). Dessa forma, percebe-se que Ulianópolis aumentou sua nota que, antes em 2015 era de 5,25, para uma média de 5,4, do IDEB (INEP, 2018).

Lourenço et al. (2017) e Rodrigues et al. (2018), que também analisaram a eficiência técnica na Educação no Estado do Pará, mas para os anos anteriores, encontraram colocações distintas das DMUs conforme apresentadas a seguir.

No trabalho do Lourenço et al. (2017), que utilizou o modelo BCC orientado ao produto, os municípios que estavam no quadro de eficiência técnica foram Afuá, Augusto Correa, Breu Branco, Moju, Portel, São Felix do Xingu, Viseu. O trabalho de Rodrigues et al. (2018), que utilizou o modelo de BCC com orientação de insumo, mostrou os seguintes municípios eficientes: Altamira, Ananindeua, Aveiro, Belém, Bom Jesus do Tocantins, Brasil Novo, Cachoeira do Arari, Jacundá, Nova Esperança do Piriá, Novo Progresso, Novo Repartimento, Placas, Rurópolis, Santarém, São Domingos do Araguaia, São Sebastião da Boa Vista, Trairão, Ulianópolis, Uruará.

Verifica-se comparativamente, com base nos dados de Rodrigues et al. (2018), que somente Santarém permaneceu eficiente, enquanto os demais municípios citados no trabalho de Lourenço et al. (2017) e de Rodrigues et al. (2018) se tornaram ineficientes. Tais municípios, como citado por Lourenço et al. (2017) e Rodrigues et al. (2018), se comparados com a base de dados de 2017, não tiveram seus insumos utilizados de forma a otimizar seus custos, alcançando seu produto eficiente, ou seja, gastou-se muito, sem produzir tanto quanto possível com os insumos que esses empregaram.

Entre os sete municípios menos eficientes, todos apresentaram rendimentos crescentes de escala, ou seja, para se tornarem eficientes, são necessárias utilizações ótimas dos insumos, para isso necessitando de algumas alterações nos seus números de docentes, de escolas, turmas e nos gastos. Assim, para que o município no ranking $116^{\circ}$, Chaves, possa aumentar sua nota média do IDEB e seus escores de eficiência, seriam necessárias reduções nos gastos com docentes, escolas e turmas, de forma que cada município permanecesse na fronteira de produção.

É importante frisar que tais resultados devem ser analisados de maneira cautelosa, uma vez que se tratam de resultados técnicos. Para que os municípios possam alcançar a eficiência, não necessariamente têm que reduzir a quantidade de professores, pois este é um fator importante para diminuir 
a baixa qualidade do Ensino Educacional. Por óbvio, são necessárias reformas coordenadas de políticas públicas nas esferas federal, estadual e municipal, para que não haja redução destes profissionais, mas, sim, seu melhor aproveitamento.

Além disso, devido ao crescente contingente populacional na sociedade, a presença de professores é imprescindível, o que pode trazer uma grande oportunidade para elevar o nível dos professores em termos de quantidade e da qualidade da educação ao longo da próxima década (BANCO MUNDIAL, 2017).

No ranking $117^{\circ}$, situa-se Anajás que, para se tornar eficiente, comparativamente a Santarém e Ulianópolis, deve reduzir a utilização do número de docentes $(440-282=158)$ e o de escolas $(102-59=43)$ e o de turmas $(490-309=181)$ e o de gastos $(34.550 .226,56-26.773 .430,56=7.776 .796,00)$.

Ainda sobre os municípios do Estado do Pará menos eficientes, o município de posição $118^{\circ}$, Cachoeira do Piriá, para se tornar eficiente, deve utilizar como referência também os municípios de Santarém e Ulianópolis e diminuir 33 docentes, 13 escolas, 16 turmas e $\mathrm{R} \$ 1.582 .057,00$. Já para os municípios no ranking $119^{\circ}$ e $120^{\circ}$, para se tornarem eficientes, será necessário utilizar como referência os mesmos municípios anteriores, enquanto o município de colocação $121^{\circ}$, Almeirim, precisa utilizar como referência somente Santarém, devendo diminuir 148 docentes, 23 escolas, 180 turmas e R\$ 6.902.612,00 gastos.

O município de Afuá, que apresentou o menor escore de eficiência $(0,5555)$, para se tornar eficiente, utilizando como referência o Abel Figueiredo, Santarém e Ulianópolis, deveria diminuir de 259, de 49, de 222 e de R\$12.500.000,00 o número de docentes, escolas, turmas e gastos, respectivamente.

Considerando os trabalhos citados, entre os municípios ineficientes para o Estado do Pará, segundo do Lourenço et al. (2017), situa-se Belém-PA, pois neste trabalho não são analisados os maiores municípios dos Estados brasileiros. Segundo Rodrigues et al. (2018), dos 100 municípios analisados em seu trabalho, 81 foram ineficientes ${ }^{6}$.

Tendo em vista os resultados do Quadro 6, nenhum dos municípios eficientes no trabalho de Lourenço et al. (2017) se apresentou como eficiente neste trabalho. No trabalho de Rodrigues et al. (2018), os municípios ineficientes permaneceram ineficientes, ou seja, não houve melhoria nos seus gastos, tendo ainda níveis de educação de baixa qualidade. Conforme a Tabela 4, esses municípios foram Afuá e Anajás.

Tendo como referência o exposto, observa-se que o município Afuá, antes eficiente, segundo Lourenço et al. (2017), mesmo com nota média do IDEB maior em 2017 $(3,0)$ do que em 2013 (2.8), foi ineficiente, o que pode estar relacionado a uma utilização excessiva dos insumos aqui utilizados e, por isso, deveria diminuir em 259 o número de docentes, em 49 o número de escolas, em 222 o número de turmas e em $\mathrm{R} \$ 12.500 .000,00$ o gasto para obtenção do máximo desempenho (IDEB) possível. Outro fator que pode estar relacionado a isto no município é que na localidade há micro escolas nas comunidades que, às vezes, ficam vazias, pela falta de alunos que migram entre as comunidades rurais durante a safra do açaí, causando evasão de alunos, prejudicando seu aprendizado, sofrendo modificações na estrutura educacional das escolas municipais de Ensino Fundamental, que funcionam no sistema regular (INSTITUTO PEABIRU E DO FUNDO VALE, 2011).

Portanto, para que essas mudanças ocorram, o gerenciamento dado ao Ensino Fundamental em relação aos gastos precisa ser revisto e reorganizado para que futuramente estes municípios ineficientes possam alcançar nível educacional satisfatório em benefício da sociedade paraense.

\subsection{Resultados do segundo estágio: determinantes de eficiência}

Os resultados aqui apresentados foram estimados utilizando considerações de Simar e Wilson (2007) e Badunenko e Tauchmann (2018), que permitem que gestores estabeleçam políticas públicas, com base nos resultados aqui apresentados, para aumentar o nível de eficiência municipal. Quadro 7 apresenta tais resultados.

Quadro 7 - Resultados do segundo estágio

\begin{tabular}{|l|c|c|}
\hline \multicolumn{1}{|c|}{ Variáveis } & Coeficiente & $\begin{array}{c}\text { Erro Padrão Robusto } \\
\text { (bootstrap) }\end{array}$ \\
\hline lnpib_pc & $0,048^{* * *}$ & 0,027 \\
\hline Lnfpm & $-0,144^{*}$ & 0,036 \\
\hline Lnpop & $0,029^{* * *}$ & 0,018 \\
\hline idhm_renda & $0,807^{*}$ & 0,247 \\
\hline Saneamento & $0,007^{* * *}$ & 0,004 \\
\hline \multicolumn{2}{|l|}{ Nota: significância estatística: ${ }^{*} 1 \% ;{ }^{* *} 5 \% ;{ }^{* * *} 10 \%}$.
\end{tabular}

Nota: significância estatística: ${ }^{*} 1 \% ;{ }^{* *} 5 \% ;{ }^{* * *} 10 \%$.

Fonte: Dados da pesquisa.

Este segundo estágio foi feito com o intuito de captar os possíveis determinantes da eficiência dos gastos públicos com Ensino Fundamental dos municípios paraenses.

Com base nos resultados do Quadro 7, percebe-se que a variável PIB per capita (lnpib_pc) se mostrou significante ao nível $10 \%$ e apresentou sinal positivo, o que era esperado, indicando que esta variável pode influenciar positivamente

6 Acará, Afuá, Água Azul do Norte, Alenquer, Almeirim, Anajás, Anapú, Augusto Corrêa, Aurora do Pará, Bagre, Barcarena, Belterra, Bragança, Branco, Breu, Breves, Cachoeira do Piriá, Canaã dos Carajás, Capitão Poço, Castanhal, Conceição do Araguaia, Concórdia do Pará, Curionópolis, Curuá, Dom Eliseu, Eldorado dos Carajás, Floresta do Araguaia, Garrafão do Norte, Goianésia do Pará, Igarapé-Miri, Ipixunado Pará, Itaituba, Itupiranga, Jacareacanga, Juruti, Limoeiro do Ajuru, Mãe do Rio, Marabá, Maracanã, Maria das Barreiras, Marituba, Medicilândia, Melgaço, Mocajuba, Moju, Nova Ipixuna, Nova Timboteua, Óbidos, Oeiras do Pará, Oriximiná, Ourém, Pacajá, Paragominas, Parauapebas, Piçarra, Ponta de Pedras, Portel, Porto de Moz, Prainha, Rio Maria, Rondon do Pará, Salinópolis, Salvaterra, Santa Bárbara do Pará, Santana do Araguaia, Santo Antônio do Tauá, São Caetano de Odivelas, São Félix do Xingu, São Francisco do Pará, São Geraldo do Araguaia, São João de Pirabas, São João do Araguaia, Senador José Porfírio, Tailândia, Terra Santa, Tomé-Açu, Tracuateua, Tucumã, Tucuruí, Viseu, Vitória do Xingu, Xinguara. 
a eficiência dos gastos dos municípios paraenses, afetando o perfil socioeconômico, podendo ser uma das causas para um melhor desempenho educacional, mas esta não é a única explicação, pois existem outros fatores para influenciar no desempenho educacional, como a diminuição da distorção idade-série, melhoria na infraestrutura das escolas, na formação e no aperfeiçoamento dos professores e na gestão das escolas. Além disto, a correlação positiva existente entre o gasto com educação e a formação do PIB municipal per capita destaca que o fator educação tem sido um diferencial tanto para o indivíduo em termos salariais, como para o crescimento do produto (INEP, 2004; SILVEIRA; TEIXEIRA, 2012).

Percebe-se que a variável $\operatorname{lnfpm}$ se mostrou estatisticamente significante em nível de $1 \%$, mas com sinal contrário (negativo). Tal resultado indica que a lnfpm está influenciando negativamente a eficiência dos gastos públicos do Ensino Fundamental no Pará, podendo este resultado estar atrelado a uma alocação dos recursos ineficiente de forma a não atender a área da educação e a população crescente, uma vez que, com esse recurso, segundo Silva (2015), o Pará, por ser um Estado com uma atividade econômica menos desenvolvida, tem um alto grau de dependência das transferências governamentais. Portanto, esta variável Infpm não está representando seu mecanismo fundamental, que é contribuir para amenizar as desigualdades regionais e para a promoção do desenvolvimento socioeconômico entre municípios (VIEIRA, 2017).

Além disso, sua influência negativa pode estar atrelada a este tipo de critério do Fundo de Participação Municipal, segundo Vieira (2017, p.4).

[...] o fundo acaba por beneficiar os municípios de pequeno porte, e não necessariamente aqueles menos desenvolvidos, contrariando, portanto, os ditames constitucionais de reduzir as desigualdades socioeconômicas.

Por sua vez, a variável lnpop foi positiva e significante em nível de $10 \%$, indicando que um aumento populacional dos municípios representa uma ampliação na eficiência dos gastos com educação que, de certa forma, pressiona os gastos da educação das pequenas cidades (SILVA; ALMEDA, 2012).

No que se refere ao Índice de Desenvolvimento Humano Municipal da esfera renda (idhm_renda), esse foi significante estatisticamente ao nível de $1 \%$, com sinal positivo, como esperado. Este resultado indica que esta é uma importante ferramenta para auxiliar nas políticas públicas dos municípios, pois pode trazer maior desenvolvimento educacional, em termos de renda, por influenciar o ingresso dos alunos no Ensino Fundamental. Por conseguinte, evidências empíricas apontam que as camadas mais ricas da população têm maior acesso à educação em detrimento das menos favorecidas. Sendo assim, esta evidência significa que parte do fracasso ou do sucesso escolar pode estar relacionada à situação socioeconômica dos alunos, não somente à ineficiência administrativa (GOMES et al., 2017).

Por fim, a variável proxy de saneamento (saneamento) foi positiva e estatisticamente significante em nível de $10 \%$. Tal resultado indica que o saneamento está influenciando positivamente a eficiência dos gastos públicos do Ensino Fundamental no Pará, isto é, o acesso à água potável, o esgotamento sanitário e a limpeza urbana proporcionados pelo Estado influenciam uma melhor educação da população (BITTELBRUNN et al., 2016).

Adicionalmente, os impactos positivos trazidos pelo saneamento para a educação nos municípios brasileiros são apresentados por Scriptore et al. (2016, p.149):

[...] um aumento marginal, em termos percentuais, da parcela da população que reside em domicílios providos de banheiro e água encanada resulta em: aumento de 0,11 pontos percentuais na taxa de frequência escolar da população de 6 a 14 anos; redução de 0,49 pontos percentuais na taxa de abandono escolar do ensino fundamental; redução de 0,96 pontos percentuais na taxa de distorção idade-série.

Dito isto, evidencia-se a necessidade de políticas públicas voltadas para o saneamento básico, pois sua falta acarreta efeitos negativos na saúde dos indivíduos, principalmente das crianças, dificultando a elas se manterem na escola, prejudicando o aprendizado e o crescimento intelectual.

\section{Conclusão}

Este estudo teve como objetivo analisar a eficiência técnica dos gastos públicos de Educação Fundamental no Estado do Pará no ano de 2017. Para isso, foi utilizada, em um primeiro estágio, a Análise Envoltória de Dados (DEA BCC) com orientação a output. Posteriormente, em um segundo estágio, foram estimados os determinantes desta eficiência.

O resultado do primeiro estágio mostrou que, dos 122 municípios analisados, apenas Abel Figueiredo, Bannach, Benevides, Santa Maria do Pará, Santarém, Sapucaia e Ulianópolis são eficientes tecnicamente, enquanto os municípios que apresentaram menores escores de eficiência foram Chaves, Anajás, Cachoeira do Piriá, Gurupá, Nova Esperança do Piriá, Almeirim e Afuá.

O resultado sobre a eficiência dos gastos dos municípios do Estado do Pará, considerando retornos constantes, foi 0,737 , e de 0,761 , considerando retornos variáveis. A eficiência de escala obteve média de 0,969 . Esses resultados mostram que os municípios do Pará necessitam ter uma melhor utilização dos insumos em relação aos gastos. Também foi observado que $53,3 \%$ da amostra apresentou rendimentos crescentes de escala, indicando que seria possível melhorar a situação em termos de eficiência, caso houvesse um crescimento na escala de produção.

No que se refere ao segundo estágio, os possíveis determinantes desta eficiência, que influenciaram positivamente, foram o PIB per capita e o IDHM renda, a população e o saneamento. Já a variável que teve o sinal negativo, mas significante, foi o FPM.

Portanto, conforme mostrado nos resultados, dos 122 municípios do Pará, houve pouco desempenho do Ensino 
Público, que pode ter sido causado pela má aplicação de recursos públicos, os quais podem não estar sendo utilizados de maneira eficiente. Estes resultados podem ser um aporte de comunicação e formulações de políticas públicas no intuito de melhorar a qualidade do Ensino Fundamental do Pará.

Os municípios do Estado do Pará que se mostraram ineficientes precisam alocar seus recursos da melhor forma possível para alcançar maior desenvolvimento, redução da desigualdade na distribuição de renda, favorecendo um maior crescimento do Estado, pois, caso continuem alocando ineficientemente seus recursos, a tendência é um menor avanço tecnológico, imensa deficiência educacional no Ensino Fundamental, sendo este principal avanço o ensino, pois é quando são iniciados os primeiros passos para a vida educacional.

Por sua vez, os prefeitos e o Governador do Estado do Pará necessitam urgentemente inserir políticas públicas para reverter esse quadro de ineficiência na Educação Fundamental, minimizando a alocação de diretores por indicação, sem exigência de nenhum nível técnico, sem capacitação, sendo este um lugar-chave de melhoria da qualidade das escolas públicas.

Diante dos resultados apresentados, os gastos dos municípios não têm sido gerenciados de forma a alcançar melhores resultados, ou seja, em sua maior totalidade, a aplicação dos gastos públicos foi ineficiente. Espera-se que este estudo leve ao aprimoramento na condução da aplicação dos recursos públicos por parte dos gestores municipais.

\section{Referências}

AFONSO, A.; ST AUBYN, M. Non-parametric approaches to education and health: expenditure efficiency in OECD countries. J. Appl. Economics. v.8, n.2, p. 227-246, 2005.

IBGE. PNAD Contínua 2016: 51\% da população com 25 anos ou mais do Brasil possuíam apenas o ensino fundamental completo. Disponível em: <https://agenciadenoticias.ibge.gov. br/agencia-noticias/2013-agencia-de-noticias/releases/18992pnad-continua-2016-51-da-populacao-com-25-anos-ou-mais-dobrasil-possuiam-apenas-o-ensino-fundamental completo.html>. Acesso em: 23 maio 2019.

ALEXANDER, N.A.; JANG, S.T. Equity and efficiency of Minnesota educational expenditures with a focus on English learns, 2003-2011: a retrospective look in a time of accountabulity. Educ. Policy Anal. Arch., v.25, n. 16, 2017.

ALMEIDA, A.T.C.; GASPARINI, C.E. Gastos públicos municipais e educação fundamental no Paraíba: uma avaliação usando DEA. Rev. Econ. Nordeste. v.42, n.3, p.621-639, 2011.

BADUNENKO, O.; TAUCHMANN, H. Simar and Wilson two-stage efficiency analysis for Stata, FAU Discussion Papers in Economics, n.8, Friedrich- Alexander-Universität ErlangenNürnberg, Inst. Economics, n.37, 2018.

BANCO MUNDIAL. Um ajuste justo: análise da eficiência e equidade do gasto público no Brasil. 2017. Disponível em: https://www.worldbank.org/pt/country/brazil/publication/brazilexpenditure-review-report

BITTELBRUNN, F. et al. Estudo da eficiência dos gastos com saneamento básico dos estados brasileiros e DF entre 2012 a
2014 por meio de análise envoltória de dados. In: CONGRESSO BRASILEIRO DE CUSTO, 23. Porto de Galinhas, 2016. Anais... Porto de Galinhas, 2016.

BRASIL. Constituição da República Federativa do Brasil. Senado Federal. Brasília - DF. Mesa, Biênio 2017-2018.

PARÁ, Governo do Estado do Pará. Instrução Normativa $n^{\circ} 3$, de 1 de março de 2016. 2016. Disponível em: <https://www. sistemas.pa.gov.br/sisleis/legislacao/3023>. Acesso em: 2 fev. 2020 .

BRASIL. Lei 5.172 de 25 de outubro de 1966. Código Tributário Nacional. Disponível em: <http://www.planalto.gov.br/ccivil_03/ LEIS/L5172.htm>. Acesso em: 9 dez. 2019.

BRASIL. Ministério da Educação. FNDE transfere R\$918 milhões da complementação da União. 2017. Disponível em: <http:// portal.mec.gov.br/ultimas-noticias/214-296700251/48061-fndetransfere-r-918-milhoes-da-complementacao-da-uniao>. Acesso em: 2 fev. 2020.

BRASIL. Ministério da Educação. Relatório Educação para Todos no Brasil, 2000-2105 / Ministério da Educação. Brasília: MEC, 2014.

BRASIL. Planalto. Disponível em: <http://www.planalto.gov.br/ ccivil_03/leis/L9394compilado.htm>. Acesso em: 17 maio 2019.

BRASIL. Ministério da Educação. Instituto Nacional de Estudos e Pesquisas Educacionais Anísio Teixeira (Inep). IDEB Índice de Desenvolvimento da Educação Básica. IDEB - Resultados e Metas. Brasília: MEC, 2018.

BRUNET, J.F.G.; BERTÊ, A.M.A.; BORGES, C.B. Estudo comparativo das despesas públicas dos estados brasileiros: um índice de qualidade do gasto público. Brasília: ESAF, 2007.

CAETANO, F.A.O.; ARAÚJO, J. A.; KHAN, A. S. Fatores condicionantes do desenvolvimento socioeconômico na América Latina: uma análise sob a perspectiva do PIB e dos indicadores globais de governança. Interações, v.20, n.1, p.95-109, 2019.

DIAS, E.J.P.; SILVA, L.M.F. Indicadores de qualidade dos gastos Públicos dos municípios da região norte do Brasil na área da educação. Rev. Periódica Fac. Belém, n.2, p.57-83, 2013.

DINIZ, J.A.; CORRAR, J.L. Análise da relação entre a eficiência e as fontes de recursos dos gastos municipais no ensino fundamental. Soc., Contabil. Gestão, v.6, n.1, p.135-149, 2011 doi: https://doi.org/10.21446/scg_ufrj.v6i1.13232

FERREIRA, G.V.; COLARES, M.L.I.S. Educação de tempo integral em Santarém: ações da Secretaria Municipal de Educação. Educ. Teor. Prática, v.27, n.56, p.457-474, 2017. doi: https://doi. org/10.18675/1981-8106.vol27.n56.p457-474

FERREIRA, C.M.C.; GOMES, A.P. Introdução à análise envoltória de dados: teoria, modelos e aplicações. Viçosa: UFV, 2009.

FNDE - Fundo Nacional de Desenvolvimento da Educação. Ministério da Educação. Disponível em: <http://www.fnde.gov. br/financiamento/fundeb/sobre-o-plano-ou-programa/sobre-ofundeb. >. Acesso em: 29 maio 2019.

FUNDAÇÃO ABRINQ. A Criança e ao Adolescente nos ODS: marco zero dos principais indicadores brasileiros. São Paulo: ABRINQ, 2017.

GOMES, A.P.; BAPTISTA, A.J.M.S. Análise envoltória de dados: conceitos e modelos básicos. In: SANTOS, M.L.; VIEIRA, W.C. (Org.). Métodos quantitativos em economia. Viçosa: UFV, 2004. p.121-160.

GOMES, A.P.; ERVILHA, G.T.; GOMES, A.P.W. Eficiência dos gastos públicos e desenvolvimento educacional nos municípios 
de Minas Gerais. In: CONGRESSO BRASILEIRO DE CUSTOS, 24. Florianópolis. Anais... Florianópolis, 2017.

GOMES, C. S. Eficiência dos sistemas municipais de educação no Estado de São Paulo. Ribeirão Preto: USP, 2010.

GREENE, W. H. Econometric analysis. New Jersey: PrenticeHall, 2002.

GREENE, W.H. On the asymptotic bias of the ordinary least squares estimator of the tobit model. Econometrica, v.49, n.2, p.505-513, 1981.

IDEB 2017. Resultados do índice de desenvolvimento da educação básica. 2017. Disponível em: 20-\%20Resumo\%20 T\%C3\%A9cnico\%20(1).pdf>. Acesso em: 20 nov. 2019.

INEP. OCDE aponta a relação desempenho $x$ PIB per capita. INEP - Instituto Nacional de Estudos e Pesquisas Educacionais Anísio Teixeira. Brasília: MEC, 2014.

INSTITUTO PEABIRU E FUNDO VALE. Diagnóstico socioeconômico, ambiental e cultural do Arquipélago do Marajó. Programa Viva Marajó, 2011.

IPEA - Instituto de Pesquisa Econômica Aplicada. Investimentos em educação e desenvolvimento econômico. Rio de Janeiro: Ipea, 1997.

LDB- Lei de diretrizes e bases da educação nacional. Brasília: Senado Federal, 2017.

LOURENÇO, R.L. et al. Eficiência do Gasto Público com Ensino Fundamental: uma análise dos 250 Maiores municípios Brasileiros. Rev. Contab. Vista Rev., v.28, n.1, p.89-116, 2017.

MACHADO JÚNIOR, S.; IRFFI, G.; BENEGAS, M. Análise da eficiência técnica dos gastos com educação, saúde e assistência social dos municípios Cearenses. Planej. Políticas Públicas, n.36, p.89-113, 2011.

MELLO, J.C.C.B.S. et al. Curso de análise de envoltória de dados. In: SIMPÓSIO BRASILEIRO DE PESQUISA OPERACIONAL, 38. 2005.

MORETTI, E. Human capital externalities in cities. Nat. Bureau Economic Res., v.4, p.2243-2291, 2004.

PRADO, L.C.D. O estado do bem-estar social na idade da razão: Célia Lessa Kerstenetzky. Cad. Desenvol., v.8, n.13, p.313-320, 2013.

ENAP. Programa Benevides à Escola. 2013. Disponível em: $<$ https://repositorio.enap.gov.br/handle/1/3617>. Acesso em: 2 fev. 2020.

RODRIGUES, M.; SILVA, D.C.C.; FERREIRA, A. L. Eficiência dos gastos em educação no Estado do Pará. Rev. Contrib. Ciênc. Soc.. 2018. Disponível em: http://www.eumed.net/rev/ cccss/2018/01/gastos-educacao-para.html

RUGGIERO, J.; VITALIANO, D. F. The efficiency of public schools using data envelopment analysis and frontier regression. Contemp. Economic Policy, v.17, n.3, p.1-33, 1999.

SANTOS, P.F. et al. A eficiência dos municípios e regiões de Minas Gerais na alocação dos gastos públicos de educação. Rev. ESPACIOS, v. 38, n. 39, 2017.

SANTOS, R.R.; FREITAS, M.M.; FLACH, Análise envoltória de dados como ferramenta de avaliação da eficiência dos gastos públicos com educação dos municípios de Santa Catarina. In: CONGRESSO BRASILEIRO DE CUSTOS, 22, 2015.

SAVIAN, M. P.G.; BEZERRA, F.M. Análise de eficiência dos gastos públicos com educação no ensino fundamental no estado do Paraná. Econ. Região, v.1, n.1, p.26-47, 2013.

SCRIPTORE, J. S. Impactos do saneamento sobre saúde e educação: uma análise espacial. São Paulo: USP, 2016.

SILVA, F.C. A importância dos fundos constitucionais na redução das desigualdades regionais no Brasil no periodo de 2001 a 2014. Natal: UFRN, 2015.

SILVA, J.L.M.; ALMEIDA, J.C.L. Eficiência no gasto público com educação: uma análise dos Municípios do Rio Grande do Norte. Planej. Políticas Públicas, n.39, p.221-244, 2012.

SILVEIRA, A.S.; TEIXEIRA, A.M.C. O efeito do gasto público na qualidade da educação. In: ENCONTRO DO ANPAD, 34. Rio de Janeiro, 2012. Anais... Rio de Janeiro, 2012.

SIMAR, L.; WILSON, P. W. Estimation and inference in twostage, semiparametric models of production processes. $J$. Econometrics, v.136, p.31-64, 2007.

SOBREIRA, R.; CAMPOS, B. C. Investimento público em educação fundamental e a qualidade do ensino: uma avaliação regional dos resultados do Fundef. Rev. Adm. Pública, p.327-346, 2008.

TRATA BRASIL. Saneamento, Saúde, Educação, Trabalho e Turismo. O Caso Santa Catarina. Rio de Janeiro. FGV/IBRE, CPS, 2008.

VIEIRA, M.A. Efeitos das transferências intergovernamentais na redução das desigualdades e na promoção do desenvolvimento socioeconômicos das regiões brasileiras. Viçosa: Universidade Federal de Viçosa, 2017.

XERFAN, A.P. Governança local em educação profissional e tecnológica: o caso do Programa Pará Profissional no Município de Ulianópolis-PA. Belém: Universidade Federal do Pará, 2018.

ZOGHBI, A.C. et al. Uma análise da eficiência nos gastos em educação fundamental para os municípios paulistas. Planej. Políticas Públicas, n.36, p.9-61, 2011.

ZOGHBI, A.C.P. et al. Mensurando o desempenho e a eficiência dos gastos estaduais fundamental e média. Estud. Econômicos, v.39, n.4, p.785-809, 2009. 